\title{
Análise de métodos de previsão de capacidade de carga em estaca raiz a partir do comportamento em ensaios de carregamento estático e dinâmico
}

Rodrigo Rogerio Cerqueira da Silva rodrigorogerio@uni9.pro.br Universidade Nove de Julho

\section{Analysis of root pile load capacity forecasting methods through the behavior between load testing static and dynamic}

\section{Análisis de métodos de previsión de capacidad de carga en estaca raíz a través del comportamiento entre ensayos de cargamento estático y dinámico}

\section{Analyse des méthodes de prévision de la capacité de charge en pieux à travers le comportement entre les tests de charge statiques et dynamiques}

\section{Resumo}

Avaliar a qualidade e o desempenho de fundações profundas é uma das maiores preocupações dentro da engenharia de fundações, sendo a carga admissível de uma estaca, estimada na fase de projeto, comprovada por meio de ensaios de carregamento estático e dinâmico. Cada ensaio possui suas peculiaridades, sendo a vantagem do ensaio estático simular a verdadeira grandeza dos carregamentos reais de uma construção e o atrativo dos ensaios dinâmicos a praticidade da análise da propagação de onda na interação por meio da resistência do solo e de parâmetros dinâmicos ao longo do fuste da estaca. O objetivo desta pesquisa é analisar, através de um programa experimental, o desempenho dos métodos mais consagrados da literatura para previsão da capacidade de carga em estacas raiz comparando ensaios de carregamento estático e dinâmico, realizados sobre a mesma estaca teste, demonstrando qual método possui melhor aplicabilidade de estimativa de carga para cada tipo de ensaio. A sequência executiva compreendeu, primeiro, a realização de carregamento dinâmico, a fim de evitar a mobilização plena da resistência das estacas, seguido de carregamento estático após um período de 20 dias, prevendo recuperar o relaxamento do solo. Considerando uma variabilidade de $\pm 20 \%$ nos resultados de previsão, observou-se que a aplicabilidade do método de Decourt (1996) para a análise da capacidade de carga, considerando os dois ensaios, apresenta maior confiabilidade quando realizado em estaca raiz, permitindo avaliar o comportamento da interação entre solo e estrutura adotados em fase de projeto.

Palavras-chave: Estaca raiz. Prova de carga dinâmica. Prova de carga estática. Métodos semiempírico.

\begin{abstract}
Assessing the quality and performance of deep foundations is one of the major concerns of geotechnical engineering, with the permissible load of a pile being estimated in the design phase proven through static or dynamic loading tests. Both tests have their peculiarities, with the advantage of static test simulating the true magnitude of the real loads of construction, and the attraction of dynamic tests due to the practicality of involving a process between wave propagation, through the interaction between soil resistance and dynamic parameters along the pile foundations. The objective of this research is to analyze, through an experimental program, the performance of the most established methods in the literature for predicting load capacity in root piles, comparing with the results between static and dynamic load tests,
\end{abstract}


carried out on the same test pile, demonstrating which method has better applicability of load estimation for each type of test. The executive sequence of the tests comprised first in carrying out dynamic loading to avoid the full mobilization of the piles resistance, followed by static loading after a period of 20 days foreseeing to recover the soil relaxation. Considering a variability of $\pm 20 \%$ in the prediction results, it was observed that the applicability of the Decourt method (1996) for an analysis of the load capacity considering the static and dynamic tests presents greater safety reliability carried out on root piles, allowing optimization of costs and solutions adopted in the project.

Keywords: Root piles. Dynamic load test. Static load test. Semi-empirical methods.

\section{Resumen}

Evaluar la calidad y el rendimiento de cimientos profundos es una de las mayores preocupaciones dentro de la ingeniería de cimientos, siendo la carga aceptable de una estaca estimada en la fase de proyecto comprobada por medio de ensayos de cargamento estático o dinámico. Ambos ensayos poseen sus peculiaridades, siendo la ventaja del ensayo estático simular la verdadera grandeza de los cargamentos reales de una construcción y el atractivo de los ensayos dinámicos por la practicidad de implicar un proceso entre propagación de onda, por medio de la interacción entre resistencia del suelo y parámetros dinámicos a lo largo del fuste de la estaca. El objetivo de este trabajo es analizar, por medio de un programa experimental, el rendimiento de los métodos más consagrados de la literatura para previsión de capacidad de carga en estacas tipo raíz, comparando con los resultados entre pruebas de carga del tipo estática y dinámica, realizados en la misma estaca test, demostrando cual de los métodos posee mejor aplicabilidad de estimativa de carga para cada tipo de ensayo. La secuencia ejecutiva de los ensayos se dio primero en la realización de cargamento estático después de un período de 20 días previendo recuperar el relajamiento del suelo. Considerando una variabilidad de $\pm 20 \%$ en los resultados de previsión, se observó que la aplicabilidad del método de Decourt (1996) para un análisis de la capacidad de carga considerando los ensayos estáticos y dinámicos, presenta mayor fiabilidad de seguridad realizado en estaca raíz, permitiendo evaluar el comportamiento de la interacción entre suelo y estructura adoptados en fase de proyecto.

Palabras-clave: Estaca raíz. Prueba de carga dinámica. Prueba de carga estática. Métodos semi-empíricos.

\section{Résumé}

L'évaluation de la qualité et de la performance des fondations profondes est une préoccupation majeure dans l'ingénierie des fondations. La charge admissible d'un pieu estimée dans la phase de conception est prouvée par des tests de charge statiques ou dynamiques. Les deux tests ont leurs particularités, l'avantage du test statique est de simuler l'ampleur réelle des charges réelles d'une construction, en tant que l'avantage des tests dynamiques est la commodité d'impliquer un processus entre la propagation des vagues, à partir de l'interaction entre la résistance du sol et les paramètres dynamique au long du fût des pieux. L'objectif de cette recherche est d'analyser, à travers un programme expérimental, la performance des méthodes de pronostic de la capacité de charge en pieux les plus établies dans la littérature. On va les comparer avec les résultats entre les tests de charge statique et dynamique, effectués sur le même pieu test. Cela peut démontrer quelle méthode a la meilleure applicabilité d'estimation de charge pour chaque type de test. La séquence exécutive des essais n'a pas été que le chargement dynamique. Cela a eu l'objectif d'éviter la pleine mobilisation de la résistance des pieux, suivi d'un chargement statique après une période de 20 jours. Ce qui a eu l'objectif de récupérer la relaxation du sol. Compte tenu d'une variabilité de $\pm 20 \%$ dans les résultats de pronostic, pour une analyse de la capacité de charge en prenant en compte des essais statiques et dynamiques, il a été observé que l'applicabilité de la méthode Decourt (1996) présente une plus grande fiabilité de sécurité réalisée sur des pieux, ce qui permet évaluer le comportement de l'interaction entre le sol et la structure adopté dans la phase de conception.

Mots-clés: Turteur racine. Test de charge dynamique. Test de charge statique. Méthodes semi-empiriques. 


\section{Introdução}

Avaliar a carga admissível de uma estaca estimada na fase de projeto é uma das maiores preocupações no campo da engenharia de fundações, comprovada somente por meio de ensaios in situ de carregamento estático ou dinâmico. Ambos os ensaios possuem suas peculiaridades, sendo a vantagem do carregamento estático simular a verdadeira grandeza dos carregamentos reais de uma estrutura, e o atrativo dos ensaios dinâmicos o fato de envolver um processo interativo entre sinais de força e velocidade medidos in loco pela resistência estática do solo e parâmetros dinâmicos obtidos ao longo do fuste da estaca.

Para pesquisadores como Bohn et al. (2017), a prova de carga é o método mais seguro para avaliar a capacidade de suporte de carga estrutural e contribuir na previsão do recalque do solo de uma fundação. Conforme Milititsk (1991), desde a alteração das propriedades do solo, ocasionada pela execução da fundação, até a falta de informações do maciço de solo, justificam a necessidade da utilização desses ensaios para produzir as condições de funcionamento a que a estaca estará submetida na realidade de forma mais próxima.

Segundo Gonçalves et al. (1996) e Silva, 2011, a definição de carga de ruptura e da carga admissível de uma estaca sob ação de carregamento estático ou dinâmico é bastante controversa, e antecede a discussão sobre a correlação entre os resultados obtidos nos dois tipos de ensaio. De acordo com os pesquisadores supracitados, a energia aplicada pelo martelo durante a cravação das estacas é insuficiente para a mobilização total do "quake" na ponta da estaca, subestimando a resistência mobilizada pela falta de penetração da estaca no solo.

De acordo com Gonçalves et al. (1996), a falta de resultados na literatura que permitam a comparação entre o desempenho das estacas em ensaios estáticos e dinâmicos está relacionada ao baixo número de ensaios estáticos condicionados até a ruptura. No Brasil, o maior número de pesquisas entre ensaio estático versus dinâmico foi realizado em estacas pré-moldadas de concreto por estudiosos como Bernardes e Nordal (1991), Bernardi et al. (1991), Niyama; Aoki (1991), Gonçalves et al. (1996) e Marchezini (2013), sendo uma contribuição do presente artigo analisar o comportamento nos dois tipos de prova de carga em estacas raiz.

É fundamental, na engenharia de fundações brasileira, adotar métodos semiempíricos baseados em dados de investigações de campo por meio de sondagens do tipo SPT e CPT, realizando uma correlação empírica-estatística em provas de carga ensaiadas até a ruptura, ocasionando incertezas na capacidade de carga, decorrentes desde a própria execução de sondagens até a falta de parâmetros técnicos, principalmente para fundações com fuste injetadas com argamassa (SILVA, 2018).

O objetivo desta pesquisa é analisar, por meio de um programa experimental, o desempenho dos métodos mais consagrados da literatura para previsão de capacidade de carga em estacas tipo raiz, comparando os resultados em provas de carga do tipo estática e dinâmica, realizados sobre a mesma estaca teste, demonstrando qual método possui melhor aplicabilidade de estimativa de carga para cada tipo de ensaio.

A sequência executiva dos ensaios compreendeu, primeiro, a realização de carregamento dinâmico, a fim de evitar a mobilização plena da resistência das estacas, seguido de carregamento estático após um período de 20 dias, prevendo a recuperação do estado natural do solo após seu relaxamento.

O tempo decorrido entre a execução da estaca e o período de realização do ensaio é um fator importante. Conforme Velloso e Lopes (2010), de acordo com as características geotécnicas do maciço de solo, durante a cravação de uma estaca podem ocorrer dois fenômenos: tanto a perda da resistência ao cisalhamento e atrito lateral quanto efeitos de recuperação da resistência do maciço ao longo do tempo. Esses fenômenos são importantes para estacas que trabalham predominantemente por atrito lateral, como as estacas raiz. Shao (2015) e Silva e Pereira (2017) verificaram que a transferência de carga das estacas injetadas é realizada no atrito lateral, garantindo uma interação radial de ancoragem pela criação do conglomerado, realizada entre argamassa e solo, aumentando a resistência à ruptura ao longo da seção do fuste das estacas injetadas.

Os resultados das provas de carga estática e dinâmica foram comparados com os cálculos obtidos nos métodos semiempíricos mais utilizados na geotecnia brasileira, como Aoki; Velloso (1975), Cabral (1986), Brasfond (1991) e Décourt (1996), utilizando parâmetros desenvolvidos especialmente para estaca raiz, 
demonstrando qual método possui melhor aplicabilidade, considerando uma variabilidade de $\pm 20 \%$ nos seus resultados para cada tipo de ensaio.

\section{Estaca raiz}

Estudos realizados por Silva (2011) demonstram que a estaca raiz surgiu na década de 50 como reforço de fundação em Nápoles, na Itália, conhecida como "palice radice". Hoje, meio século depois, tem tido larga aplicação como elemento de fundações especiais. Foi introduzida no Brasil a partir de meados da década de 70, empregada em obras que necessitavam de reforços modernos e eficientes para prédios e monumentos antigos sujeitos, por diferentes razões, à ocorrência de recalques. Segundo Silva e Pereira (2017), o conceito inicial era produzir um sistema de fundação com grupos de estacas inclinadas em várias direções, consolidando o maciço de solo, como o sistema de raízes de uma árvore (Figura 1).

Figura 1 - Catedral, Agrigento (Itália), reforçada na base com estaca raiz

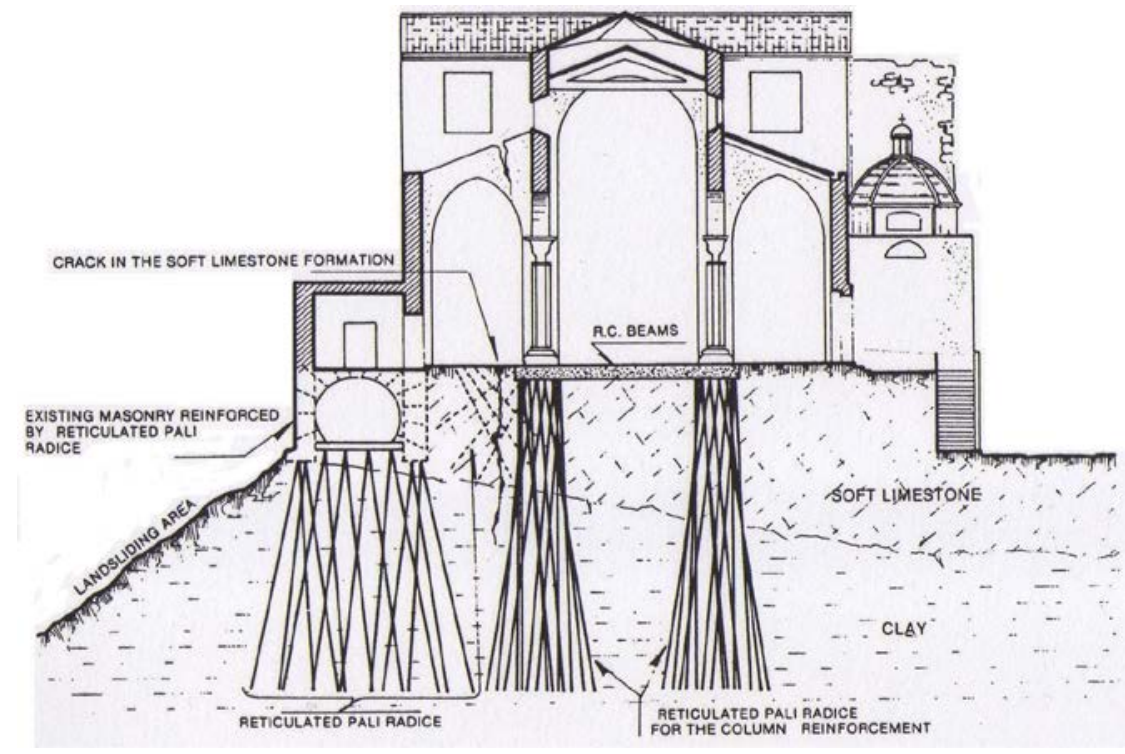

Fonte: Silva e Pereira (2017).

A denominação estaca escavada injetada inclui vários tipos de estacas moldadas in loco, por meio de diferentes técnicas. A ABNT-NBR 6122 (ASSOCIAÇÃO BRASILEIRA DE NORMAS TÉCNICAS, 2019) define como estaca raiz a fundação executada por perfuração rotativa ou rotopercussiva, revestida integralmente no trecho em solo por um conjunto de tubos metálicos recuperáveis, injetada com argamassa para moldar o fuste. A injeção ascendente deve ser interrompida apenas quando a argamassa extravasar limpa, sem presença de lama ou detritos de solo, devendo o tubo ser preenchido até que atinja a superfície do terreno. Ao final da injeção da estaca, durante a retirada dos tubos e revestimentos, aplica-se pressão no topo da estaca com ar comprimido, em pressões inferiores a 0,5 MPa, o que permite garantir a integridade e alargamento do diâmetro da estaca. A Figura 2 demonstra a execução de uma estaca raiz. 
Figura 2 - Processo executivo de estaca raiz injetada com argamassa

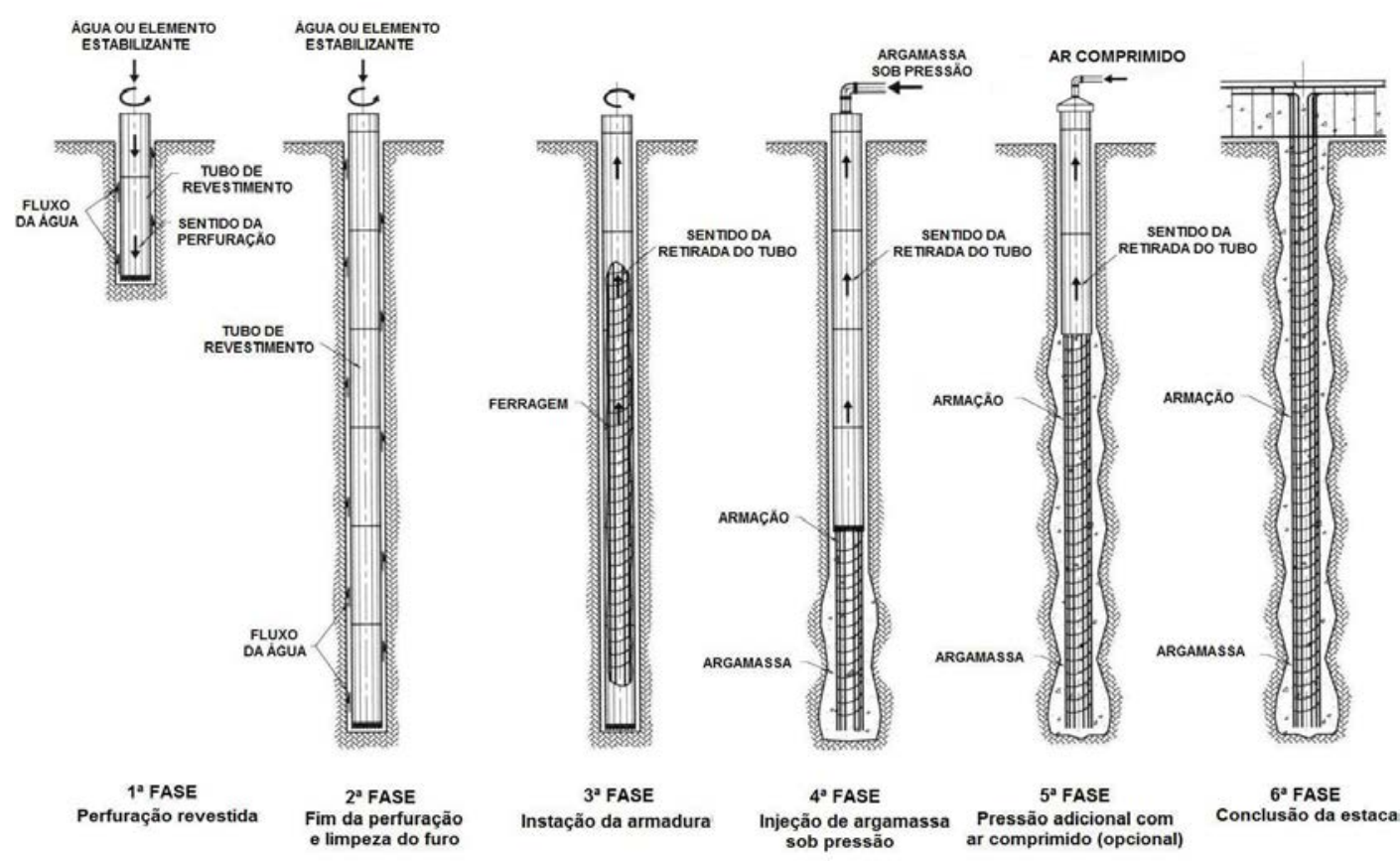

Fonte: Silva (2011).

Em comparação com as demais estacas injetadas, a estaca raiz possui vantagens técnicas ao atravessar camadas de solo e rocha, sendo consagrada em obras geotécnicas encontradas na literatura, como: estabilidade de encostas em taludes instáveis, aumentando a resistência à tração e ao cisalhamento no caso de ocorrências de escorregamentos (HUANG; FANG, 2013); execução em túnel para estabilidade na abóboda, com o intuito de minimizar recalques e proporcionar menores deslocamentos nas medidas internas de convergência na seção do maciço (MELO, 2013); reforço de fundação realizada praticamente sem barulho ou vibração, com vantagem do furo revestido, não causando descompressão do terreno (HEMEDA; BONINO, 2014); fundação predial, devido a sua elevada capacidade de carga (SILVA; PEREIRA, 2017).

Durante a execução das estacas injetadas, pesquisadores como Schlosser e Frank (2004), Shao (2015), Silva (2017) e Silva (2018) verificaram que injeções de argamassa por meio de alta pressão provocam modificações nas propriedades mecânicas dos solos coesivos, como a resistência ao cisalhamento e compressão axial, e nos parâmetros físicos, como índice de vazios e coeficiente de permeabilidade.

\section{Materiais e métodos}

O programa experimental foi desenvolvido por meio da técnica de investigação geotécnica de sondagem à percussão (SPT) e de ensaios estáticos e dinâmicos, em duas estacas do tipo raiz, realizando a previsão da capacidade de carga dos métodos brasileiros específicos para estaca raiz. A seguir, são detalhadas: as características do maciço de solo, a execução das estacas de teste, os ensaios estáticos e dinâmicos, e os métodos para previsão da capacidade de carga das estacas.

\subsection{Características geológicas e geotécnicas}

A avaliação da capacidade de carga das estacas foi realizada no município de Arujá, região nordeste da Região Metropolitana de São Paulo. De acordo com Perrotta et al. (2005), o substrato geológico do município é caracterizado por rochas oriundas de granito e gnaisses de baixo a médio grau, com predomínio, na região oeste, dos solos de classe terciária. Foram executadas quatro sondagens do tipo SPT, demonstrando que o 
subsolo local das estacas de teste é constituído por uma camada superficial de solo residual, com espessura de $2 \mathrm{~m}$, com textura variando entre argila siltosa pouco arenosa e silte arenoso pouco siltoso, de consistência mole e cor marrom, e que, sotoposta a essas camadas, encontra-se uma transição de camada residual de $18 \mathrm{~m}$, de textura silto-argilosa, com presença de mica e cascalho de cor avermelhada. A camada inferior é resultante da alteração da rocha local proveniente de um metamorfismo, na qual predominam micaxistos, associados a algumas intrusões de quartzitos, com espessura de $4 \mathrm{~m}$ e de cor amarelada. A Figura 3 apresenta um perfil com as características encontradas nas sondagens NSPT executadas em cada bloco de fundação das estacas raiz.

Figura 3 - Perfil esquemático do solo com SPT próximo às estacas executadas

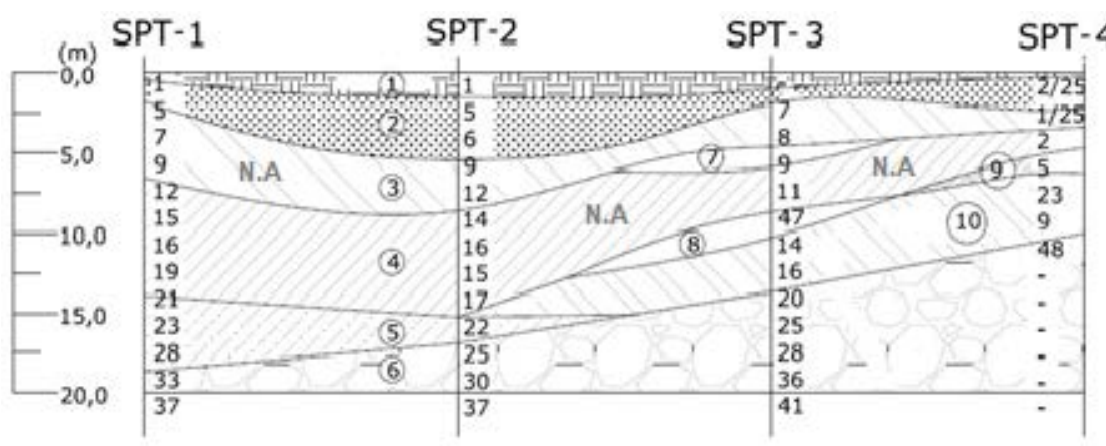

1 - ARGILA SILTOSA MUITO MOLE

2 - SILTE ARENOSO PQUCO COMPACTO

3 - SILTE ARENOSO MED. COMPACTO

4 - SILTE ARENOSO COMPACTO

5 - SILTE ARENOSO MUITO COMPACTO

6 - SOLO DE ALTERAÇÄO DE ROCHA

7 - SILTE ARGILOSO

8 - CASCALHO

9 - ARGILA SILTOSA MUITO MOLE

10 - ARGILA SILTOSA MOLE

Fonte: Autor (2020).

Na Tabela 1 encontram-se os principais índices físicos dos solos de acordo com a profundidade, considerando como critério de parada para caracterização a profundidade limite da fundação. Esses parâmetros foram obtidos em amostras de solo amolgadas conforme a realização das sondagens.

Tabela 1 - Índices físicos do local dos blocos de fundação das estacas do tipo raiz

\begin{tabular}{c|c|c|c|c|c|c|c}
\hline $\begin{array}{c}\text { Profundidade } \\
(\mathrm{m})\end{array}$ & $\mathrm{e}$ & $\mathrm{n}$ & $\mathrm{rs}\left(\mathrm{kN} / \mathrm{m}^{3}\right)$ & $\mathrm{Sr}$ & \multirow{2}{*}{$\mathrm{LP}$} & $\mathrm{LL}$ & $\mathrm{w}$ \\
\cline { 7 - 8 } & & & $\%$ & & $(\%)$ \\
\hline 1 & 1,171 & 53,75 & 27,74 & 40,28 & 26,5 & 35 & 16,3 \\
\hline 2 & 1,088 & 51,99 & 27,74 & 43,78 & 20,9 & 31,4 & 16,8 \\
\hline 3 & 0,956 & 48,61 & 27,73 & 53,98 & 18,6 & 38 & 18,9 \\
\hline 4 & 0,639 & 35,03 & 27,55 & 47,97 & 19,4 & 34,6 & 19,6 \\
\hline 5 & 0,976 & 49,49 & 27,70 & 49,9 & 21,9 & 30 & 23,2 \\
\hline 6 & 1,301 & 56,34 & 28,10 & 49,75 & 25,8 & 35,6 & 31,1 \\
\hline 7 & 1,081 & 52,95 & 28,65 & 55,84 & 25,9 & 33,4 & 34,9 \\
\hline 8 & 1,103 & 52,58 & 29,85 & 61,31 & 23 & 38,2 & 32,7 \\
\hline 9 & 1,391 & 58,19 & 29,08 & 63,19 & 22,9 & 40 & 37,6 \\
\hline 10 & 1,278 & 53,91 & 29,02 & 52,74 & 19,3 & 36 & 34,7 \\
\hline 11 & 1,307 & 57,38 & 28,30 & 55,98 & 21,4 & 37,9 & 33,18 \\
\hline 12 & 1,239 & 58,62 & 29,73 & 59,69 & 20,1 & 33,2 & 32 \\
\hline 13 & 1,281 & 52,48 & 28,70 & 53,49 & 25,5 & 31 & 27,18 \\
\hline 14 & 1,383 & 54,30 & 28,99 & 54,3 & 24 & 30 & 29 \\
\hline Média & 1,157 & 52,54 & 28,49 & 53,01 & 22,5 & 34,6 & 27,7 \\
\hline
\end{tabular}

Onde: índice de vazios (e); porosidade (n), peso específico das partículas sólidas ( $r s)$, grau de saturação (Sr), limite de plasticidade (LP), limite de liquidez (LL), teor de umidade (w).

\subsection{Características das estacas de teste}

As estacas raiz, executadas como fundação para uma obra de infraestrutura, tinham tubo de revestimento com diâmetro externo de $410 \mathrm{~mm}$ e comprimento de $14 \mathrm{~m}$. Concluída a perfuração, foi posicionada junto ao 
fuste a armadura, constituída por seis barras de aço CA50, com diâmetro de $16 \mathrm{~mm}$, e estribos, com diâmetro de $6,3 \mathrm{~mm}$, posicionados a cada $20 \mathrm{~cm}$. Para injeção de argamassa, foi considerado um traço de 1:2,36, relação água/cimento de 0,6 , com massa unitária de $2.100 \mathrm{~kg} / \mathrm{m}^{3}$ e consumo de cimento de $480 \mathrm{~kg} / \mathrm{m}^{3}$. Para a injeção das estacas de teste, denominadas ER-01 e ER-02, foi realizada a aplicação de golpe de ar a cada retirada de 2 metros de revestimento, com pressão de $10 \mathrm{kgf} / \mathrm{cm}^{2}$, a fim de adensar a argamassa e comprimila contra o solo. Durante a preparação das argamassas junto aos misturadores, foram moldados corpos de prova com dimensões de $50 \times 100 \mathrm{~mm}$, submetidos a ensaios resistência à compressão nas idades de 3,7 e 28 dias. A Tabela 2 demonstra às características de resistência a compressão das argamassas injetadas durante a execução das estacas raiz, superando em $30 \%$ o valor determinado em projeto.

Tabela 2 - Características das argamassas das estacas ensaiadas

\begin{tabular}{c|c|c|c|c}
\hline \multirow{2}{*}{ Estaca raiz } & \multirow{2}{*}{$\left(f_{c}\right)$ determinado em projeto aos 28 dias (MPa) } & \multicolumn{3}{|c}{$\left(f_{c}\right)$ determinado em ensaio de compressão simples (MPa) } \\
\cline { 3 - 5 } & & 3 dias & 7 dias & 28 Dias \\
\hline ER-01 & \multirow{2}{*}{25} & 15,2 & 23,2 & 35,2 \\
\hline ER-02 & & 15,6 & 22,4 & 34,1 \\
\hline
\end{tabular}

\subsection{Provas de carga estática (PCE) e dinâmica (PDA)}

Para as provas de carga estática (PCE), foi considerado o carregamento lento, de acordo com a ABNTNBR 12131 (2006), com fator de segurança igual a 2,0, sendo os estágios de carregamento e descarregamento calculados em função da carga de trabalho da estaca (130 tf).

Para obter a reação necessária, foram executadas quatro estacas raiz, situadas em torno da estaca de teste, servindo de apoio para uma viga de reação secundária em duplo I soldada (perfil metálico). O sistema de reação principal é formado por uma viga metálica, em duplo I, travada por barras de aço complementar (DYWIDAG), introduzido no interior das estacas que auxiliaram no travamento do sistema de reação secundário com a utilização de porcas e placas.

Abaixo das vigas de reação encontra-se uma célula de carga elétrica de $500 \mathrm{tf}$, provido de um painel para leituras, engastada a um macaco hidráulico com capacidade de $500 \mathrm{tf}$, alimentados por uma bomba hidráulica elétrica. Em cada estágio, a carga aplicada era controlada com precisão pela célula, sendo medidas as deformações da cabeça da estaca e realizadas as leituras de deformação por quatro extensômetros analógicos com precisão de $0,01 \mathrm{~mm}$ e curso de $100 \mathrm{~mm}$. A Figura 4 representa, de forma geral, o esquema adotado na utilização das provas de carga à compressão.

Figura 4 - Perfil esquemático do sistema de reação das estacas de teste para ensaio de carregamento estático

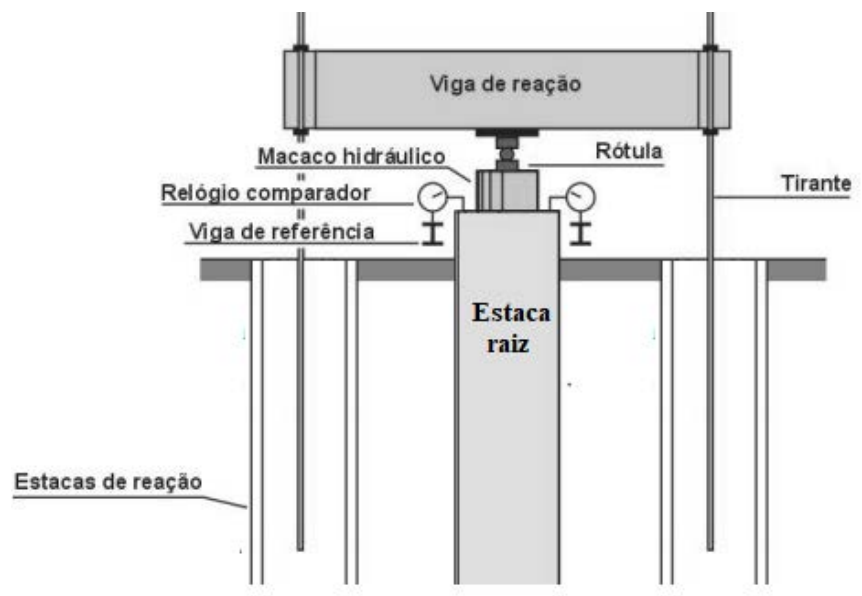

Fonte: Autor (2020). 
A Figura 5 (a) e (b) apresenta o gráfico de carga $x$ deslocamento $(\mathrm{mm})$, obtido a partir das provas de carga à compressão para as estacas raiz ER01 e ER-02 ensaiadas.

Figura 5 - Curvas carga $x$ deslocamento fornecidas pelas provas de carga estática (PCE), sendo (a) estaca E01 e (b) estaca E02

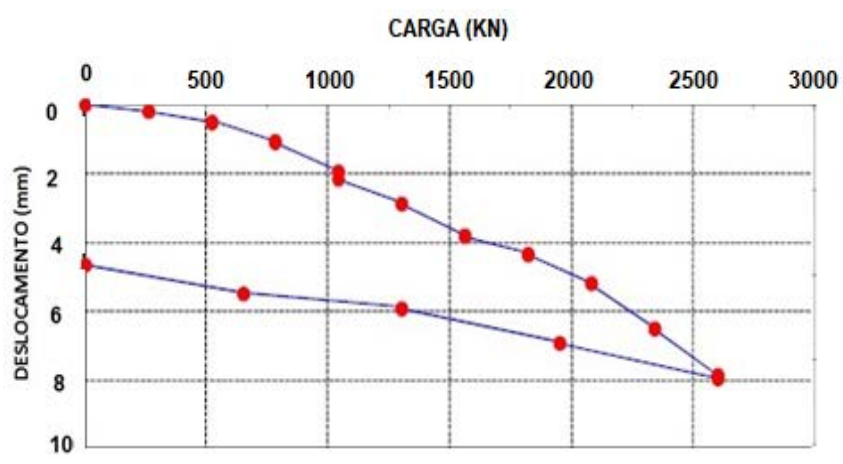

(a)

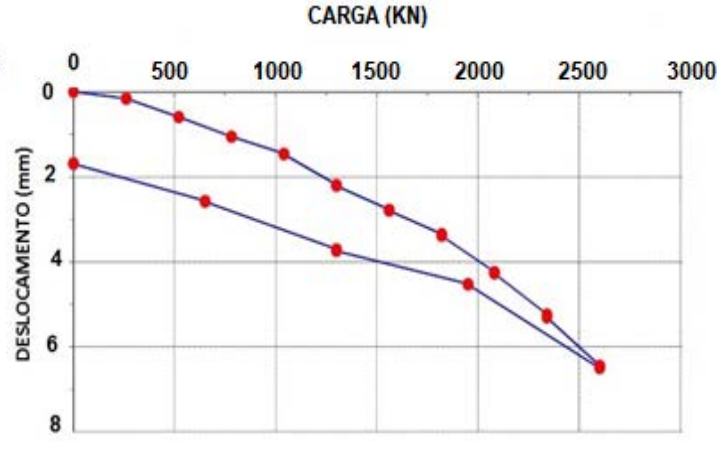

(b)

Para a realização dos ensaios dinâmicos, foi utilizado equipamento modelo PDI, composto por um analisador de cravação de estacas e um conjunto de sensores formado por um par de strain gages (sensibilidade entre 350 e $450 \mu \mathrm{s} / \mathrm{m} \mathrm{V} / \mathrm{V}$ ), e um par de acelerômetros piezelétricos com amplificador acoplado (sensibilidade de $1 \mathrm{~m} \mathrm{~V} / \mathrm{g}$ ). Para utilização de cravação das estacas, foi utilizado um bate-estaca equipado com martelo para queda livre, com massa de 2,60 tf. A interpretação dos resultados obtidos nos ensaios dinâmicos foi realizada pelo método CAPWAP (Case Pile Wave Analysis Program), utilizado para previsão da capacidade de carga por meio da análise interativa dos dados de medições de força e aceleração em função do deslocamento da estaca e reação dos parâmetros elásticos do maciço de solo. A Tabela 3 apresenta um resumo dos números de golpes realizados durante os ensaios.

\section{Tabela 3 - Resultado das análises CAPWAP}

\begin{tabular}{l|c|c|c}
\hline \multirow{2}{*}{ Estaca } & Martelo & \multirow{2}{*}{ Número de golpes } & Alturas de queda do martelo \\
\cline { 2 - 2 } & $(\mathrm{kN})$ & & $(\mathrm{cm})$ \\
\hline ER-01 & 260 & 3 & $20 / 50 / 70$ \\
\hline ER-02 & 260 & 3 & $20 / 50 / 70$ \\
\hline
\end{tabular}

Na tabela 4 são apresentados os principais resultados obtidos através das provas de carga dinâmica (PDA) para as grandezas de maior interesse, obtidos através das análises efetuadas pelo programa CAPWAP

Tabela 4 - Resultado das análises CAPWAP

\begin{tabular}{|c|c|c|c|c|c|c|c|}
\hline \multirow[t]{2}{*}{ Estaca } & \multirow{2}{*}{$\frac{\text { Resistência total }}{(\mathrm{kN})}$} & \multicolumn{2}{|c|}{ Resistência lateral } & \multicolumn{2}{|c|}{ Resistência de ponta } & \multirow{2}{*}{$\frac{\text { Quake lateral }}{(\mathrm{mm})}$} & \multirow{2}{*}{$\frac{\text { Quake de ponta }}{(\mathrm{mm})}$} \\
\hline & & $(\mathrm{kN})$ & $(\%)$ & $(\mathrm{kN})$ & $(\%)$ & & \\
\hline ER-01 & 2360 & 13990 & 59 & 9610 & 41 & 1,29 & 2,14 \\
\hline ER-02 & 2450 & 15730 & 64 & 8770 & 36 & 1,02 & 1,76 \\
\hline
\end{tabular}

A eficiência do sistema de cravação e integridade das estacas e as propriedades dinâmicas do solo foram obtidas por meio do programa CAPWAP, cujo sinal obtido de velocidade calcula-se baseado em um modelo assumido do solo a partir da criação de uma curva de força em função do tempo, sendo que o modelo é aprimorado até que se obtenha o melhor ajuste entre as curvas de força medida e força calculada. Com base nos ensaios dinâmicos, foram avaliadas as capacidades de carga das estacas raiz em dois blocos de fundação utilizando-se o modelo CAPWAP, e foram obtidos os dados das curvas carga x recalque, conforme Figura 6. 
Figura 6 - Curvas carga $\mathrm{x}$ deslocamento obtidas pelo programa CAPWAP para as estacas (a) ER-01 e (b) ER-02

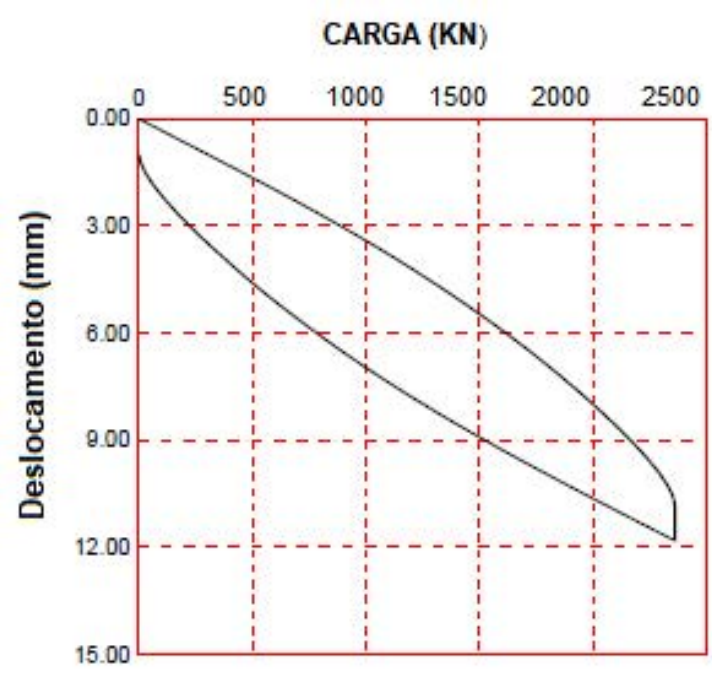

(a)

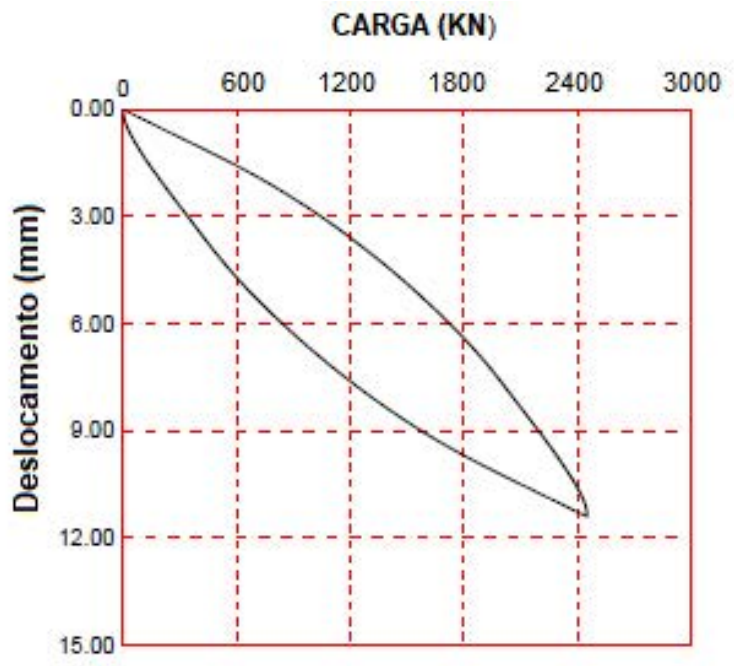

(b)

\subsection{Métodos semiempíricos}

O número restrito de contribuições existentes na literatura brasileira para previsão de carga em estaca raiz motivou a utilização de métodos baseados em valores estáticos, fornecidos por meio de ensaios do tipo cone penetration test (CPT), ou valores dinâmicos, obtidos em sondagens à percussão (SPT). Esses métodos empregam condições específicas para determinado tipo de fundação, limitando sua determinação de capacidade de carga de acordo com a geologia local e seu método construtivo.

A previsão da capacidade de carga foi realizada através dos métodos mais consagrados no meio geotécnico, como Aoki; Velloso (1975), Cabral (1986), Brasfond (1991) e Decourt (1996), considerando especificamente os parâmetros empregados para estacas injetadas. Para o método de Cabral (1986), foi considerada uma pressão de injeção de argamassa em sua equação de $10 \mathrm{kgf} / \mathrm{cm}^{2}$. Já no método de Decourt (1996), para a estimativa da capacidade de carga, foram utilizados os coeficientes de determinação das parcelas de resistência lateral específicas para estaca raiz.

\section{Resultados e discussão}

\subsection{Previsão da capacidade de carga}

A determinação da capacidade de carga das estacas foi realizada a partir do diâmetro de projeto (410 $\mathrm{mm}$ ), por conta da variação de diâmetro das estacas após a aplicação de golpes de ar durante sua execução. Como parâmetros de índice de resistência à penetração $\mathrm{N}_{\mathrm{SPT}}$, foram utilizadas as sondagens executadas junto as estacas testes, além da utilização dos parâmetros estabelecidos pela literatura para estaca raiz na previsão de capacidade de carga. A Tabela 5 apresenta os valores de previsão de capacidade de carga estimados pelos métodos semiempíricos. 
Rodrigo Rogerio Cerqueira da Silva

Tabela 5 - Previsões das capacidades de carga pelos métodos semi-empíricos

\begin{tabular}{l|c|c|c|c}
\hline Estaca Raiz & $\begin{array}{c}\text { Aoki e Velloso } \\
(\mathrm{kN})\end{array}$ & $\begin{array}{c}\text { Decourt } \\
(\mathrm{kN})\end{array}$ & $\begin{array}{c}\text { Brasfond } \\
(\mathrm{kN})\end{array}$ & $\begin{array}{c}\text { David Cabral } \\
(\mathrm{kN})\end{array}$ \\
\hline E01 & 6000 & 17500 & 11600 & 13500 \\
\hline E02 & 8500 & 23000 & 15600 & 18800 \\
\hline Média & 7250 & 20250 & 13600 & 16150 \\
\hline Desvio Padrão & 17,68 & 38,89 & 28,28 & 37,48 \\
\hline Coeficiente de variação & 0,24 & 0,19 & 0,21 & 0,23 \\
\hline
\end{tabular}

As bibliografias de engenharia de fundações que analisam a previsão para capacidade de carga consideram os valores inferidos aceitos quando apresentarem a variabilidade $\pm 20 \%$ da carga de ruptura. A Figura 7 apresenta o desempenho dos métodos semiempíricos comparando as cargas obtidas in loco nas provas de carga estática (PCE) e dinâmica (PDA).

Figura 7 - Comparação entre os valores de estimativa de capacidade de carga pelos métodos semiempíricos e as carga de ensaio PCE e PDA

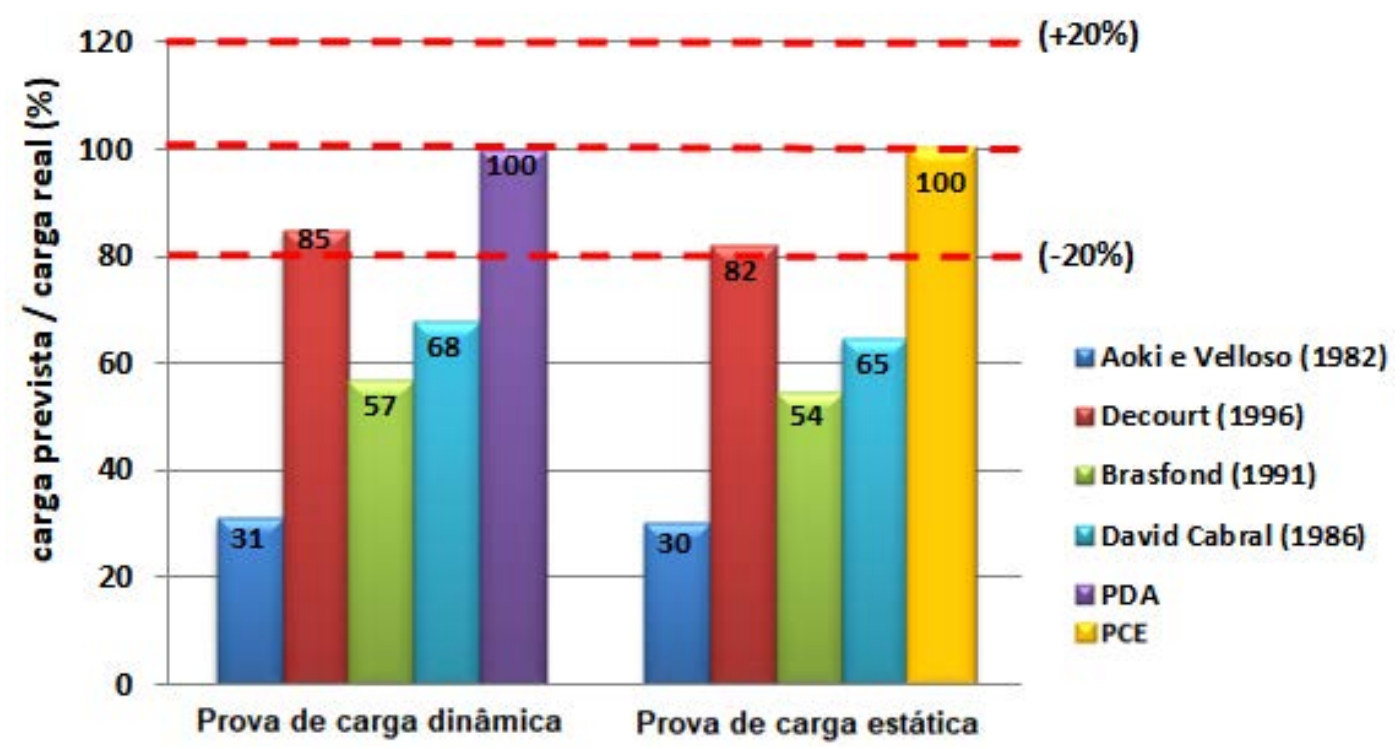

Fonte: Autor (2020).

Considerando a média dos resultados obtidos in loco pelos ensaios de carregamento estático e dinâmico, assim como a média dos valores encontrados pelos métodos semiempíricos, o que apresentou valores mais próximos da realidade, conforme Figura 6, foi Décourt (1996), subestimando a carga média das estacas em 15\% para PDA e 18\% para PCE. O desvio padrão e o coeficiente de variação dos erros apresentam discrepâncias entre os quatro métodos, porém o método de Décourt (1996) apresenta menor variabilidade na distribuição dos resultados, demonstrando ser o método mais confiável ao considerar os parâmetros específicos para estaca raiz em sua previsão de carga. Estudos realizados por Moura (2011), Silva e Pereira (2017) e Silva (2018) também demonstraram que o método de Decourt (1996) é capaz de prever a capacidade de carga para estaca raiz, chegando atingir entre $70 \%$ a $80 \%$ do valor de referência obtido pelas provas de carga.

A discrepância em subestimar a capacidade de carga a partir dos métodos semiempíricos para estacas do tipo raiz está relacionada com a metodologia de execução, diferenciada em relação às estacas escavadas tradicionais. Schlosser e Frank (2004) e Silva 2017) demonstram que o efeito de melhoria da capacidade de carga de ruptura das estacas tipo raiz é influenciado pela pressão de injeção, fenômeno que ocorre nos solos de alto módulo de deformação, sejam arenosos, sejam argilosos. O alargamento do fuste, ocasionado pelo 
aumento da pressão de injeção nas estacas do tipo raiz, está relacionado com as mudanças dos parâmetros físicos e mecânicos do maciço do solo circundante das estacas de teste.

Os índices físicos dos solos das estacas ensaiadas, encontrados na Tabela 1, contribuem para o aumento do diâmetro da estaca devido à compactação radial do solo, ocasionado pelo volume e pressão da argamassa injetada, fenômeno que ocorre nos solos com maiores valores de índices de vazios e porosidade. Parâmetros como índices físicos não são considerados pelos métodos de previsão de carga semiempíricos, além de parâmetros específicos para estaca raiz, aumentando a disparidade com os valores encontrados in loco.

\subsection{Comportamento em PCE e PDA}

Após a aplicação do último estágio de carga estática (260tf), as deformações estabilizaram em 16 horas para a estaca ER-01 e 17 horas para a estaca ER-02. De acordo com as curvas apresentadas, verifica-se que as estacas ensaiadas, no seu último estágio de carga, apresentaram valor de deformação de 7,96 mm para estaca ER-01 e de 6,51 mm para estaca ER-02, equivalente a 1,95\% e 1,58\% de seu diâmetro nominal de $410 \mathrm{~mm}$, respectivamente. Após o descarregamento, por meio da curva carga $\mathrm{x}$ recalque, foi verificado um recalque considerado residual para a estaca ER-01, de 4,63 mm, e 1,62 mm para E02, ou seja, 1,12\% e $0,40 \%$ do diâmetro da estaca, respectivamente.

A ruptura convencional na literatura geotécnica correspondente a uma deformação do topo ou ponta da estaca em $10 \%$ do seu diâmetro para estacas escavadas executadas em solos argilosos. Assim, para as estacas de teste em estudo, a ruptura convencional seria de $41 \mathrm{~mm}$. Entretanto nota-se que a curva carga $\mathrm{x}$ recalque não apresentou ruptura convencional bem definida para nenhum tipo de prova de carga, sendo esse tipo de comportamento característico para estacas injetadas com argamassa executadas em solos coesivos.

As provas de cargas estáticas permitem simular de modo mais próximo da realidade o comportamento da fundação através da interação com solo, através da aplicação de cargas em níveis crescentes, até atingir a carga limite do ensaio, ou até a completa ruptura do sistema de reação, o escoamento do solo. A proximidade da capacidade de carga das estacas e dos valores de recalque residuais encontrados entre os dois tipos de ensaio comprovam a confiabilidade e substituição para capacidade de resistência estática em fundações, em específico a estaca raiz, em ensaios dinâmicos.

As diferenças encontradas para a capacidade de carga final entre o ensaio dinâmico e estático é de 2\% para estaca ER-01 e 4\% para estaca ER-02, ou seja, praticamente nulas ao se comparar aos limites estabelecidos na literatura, conforme sugerido por Gonçalves et al. (2000), cuja diferença entre os dois tipos de prova de carga deve se encontrar em torno de $20 \%$.

As energias de cravação foram suficientes para penetrar as estacas e mobilizar as parcelas de resistência de ponta e lateral. A partir dos dados de ensaio de carregamento dinâmico, obtidos pela análise CAPWAP, foi possível verificar os valores máximos de deformação elástica do solo na ponta da estaca raiz, variando entre 2,14 e 1,76 para as estacas ER-01 e ER-02, respectivamente.

Segundo estudos de Bernardes e Nordal (1991), o comportamento da carga de ruptura, obtido através de ensaios dinâmicos, aproxima-se da capacidade de carga estática somente quando a energia de cravação das estacas realizada pelo martelo de impacto for suficiente para provocar a penetração da ponta da estaca, comportamento obtido para as estacas de teste, com quake de ponta maior que o lateral.

\section{Conclusões}

As análises realizadas na presente pesquisa demonstram que os resultados obtidos pelos métodos mais utilizados na literatura para previsão de capacidade de carga subestimam os valores obtidos pelos ensaios de controle de qualidade realizados in loco nos ensaios de carregamento estático e dinâmico, entre $20 \%$ a $70 \%$. O método de Decourt (1996) foi o único que apresentou melhor estimativa da capacidade de carga e menor 
dispersão dos resultados obtidos in loco, levando em consideração os parâmetros e métodos específicos para estacas do tipo raiz.

A distância dos valores entre as formulações para estimativa da capacidade de carga e provas de cargas, está relacionada com a influência do consumo de argamassa e a pressão de injeção realizada durante a execução, alterando a previsão de carga de ruptura dos métodos devido ao grau de fraturamento ou do maciço de solo. O aumento do diâmetro ao longo do fuste, pela pressão de injeção, proporciona protuberâncias e irregularidades, aumentando a interação entre estaca e solo, contribuindo com o atrito lateral.

As provas de carga dinâmica contribuem para demonstrar o comportamento real da estrutura na interação solo-estaca quando comparadas com ensaio de carregamento estático pelo comportamento da curva carga $\mathrm{x}$ recalque. A energia de ensaio aplicada pelo carregamento dinâmico demonstra que os deslocamentos gerados pela estaca possuem maior parcela de resistência lateral, representando o comportamento da estaca raiz, que trabalha por atrito lateral. $O$ ensaio de carregamento dinâmico representa uma alternativa para verificar o verdadeiro comportamento das estacas in loco devido a sua praticidade e rapidez na execução, contribuindo para a confiabilidade de resultados satisfatórios, visando à substituição de ensaios estáticos.

A realização de ensaios dinâmicos e estáticos na mesma estaca deverá respeitar o tempo de cicatrização do solo, ou seja, o reensaio deverá possibilitar o aumento da rigidez do solo para contribuir com o aumento das parcelas do atrito lateral e da ponta da estaca.

As conclusões apresentadas na presente pesquisa se referem às estacas de teste executadas em maciço de solo de acordo com as características geológicas e geotécnicas apresentadas, sendo necessário o crescimento constante de banco de dados de ensaios entre provas de cargas estática e dinâmica aplicadas em estaca do tipo raiz, executadas em diferentes tipos de solo, ao qual permitirá maior confiabilidade no emprego de métodos semiempíricos de previsão de capacidade de carga.

\section{Referências}

ASSOCIAÇÃO BRASILEIRA DE NORMAS TÉCNICAS. ABNT NBR 12131: estacas: prova de carga estática. Rio de Janeiro: ABNT, 2006.

ASSOCIAÇÃO BRASILEIRA DE NORMAR TÉCNICAS. ABNT NBR 6122: projeto e execução de fundações. Rio de Janeiro: ABNT, 2019.

AOKI, N; VELLOSO, D. A. An aproximated method to estimate the bearing capacity of piles. In: PANAMERICAN CONFERENCE ON SOIL MECHANICS AND FOUDANTION ENGINEERING, 5., 1975, Buenos Aires. Procedings [...]. Buenos Aires: [s. n.], 1975. p. 367-376.

BERNARDES, G. P.; NORDAL, S. Estudo da capacidade de carga de estacas modelo através de ensaios estáticos e dinâmicos. In: SEMINÁRIO DE ENGENHARIA DE FUNDAÇÕES ESPECIAIS, 2., 1991, São Paulo. Anais [...]. São Paulo: ABMS, 1991. p. 17-30.

BERNARDI, E.; CAMPOS, G. C.; MACHADO, J. R. A. O método CAPWAPCE e sua aplicação no caso do campo experimental da EPUSP/ABEF. In: SEMINÁRIO DE ENGENHARIA DE FUNDAÇÕES ESPECIAIS, 2., 1991, São Paulo. Anais [...]. São Paulo: ABMS, 1991. p. 31-39.

BOHN, C; LOPES, A. S; FRANK, R. Development of axial pile load transfer curves based on instrumented load tests. Journal of Geotechnical and Geoenvironmental Engineering, [S. I.], v. 143, n. 1, p. 04016081-04016081, 2017.

BRASFOND FUNDAÇÕES ESPECIAIS S.A. Catálogo. São Paulo: Brasfond, 1991. 38p.

CABRAL, D. A. O uso de estacas raiz como fundação de obras normais. In: CONGRESSO BRASILEIRO 
DE MECÂNICA DOS SOLOS E ENGENHARIA DE FUNDAÇÕES, 8., 1986. Anais [...]. Porto Alegre: [s. n.], 1986. v. 6, p. 71-82.

DÉCOURT. L. A ruptura de fundações avaliada com base no conceito de rigidez. In: $3^{\circ}$ SEMINÁRIO DE ENGENHARIA DE FUNDAÇÕES ESPECIAIS E GEOTECNIA, 3., 1996. Anais [...]. São Paulo: [s. n.], 1996. v. 20, 10p.

GONÇALVES, C.; ANDREO, S. C.; BERNARDES, G. P. Prova de carga dinâmica. São Paulo: [s. n.], 1996.

GONÇALVES, C.; ANDREO, C. S.; BERNARDES, G. P.; FORTUNATO, S. G. S. Controle de fundações profundas através de métodos dinâmicos. 1. ed. São Paulo: [s. n.], 2000.

HEMEDA, S.; BONINO, V. U. Paliradice (Root pile, Micro pile) technology for preservation of historical islamic monuments in Cairo Egypt. International Journal of Advanced Engineering and Science, Tamil Nadu, v. 3, n. 2, 2014.

HUANG, Y; FANG, A. application of root piles in the slope reinforcement on northern shore of Qingxiu lake. Western China Communications Science \& Technology, [S. I.] v. 7, 2013.

MARCHEZINI, S. F. Comparação entre métodos estáticos e dinâmicos de previsão de capacidade de carga em estacas assentes em solo tropical. Orientador: Wilson Conciani. 2013. 171f. Dissertação (Mestrado em Geotecnia) - Universidade Estadual de Brasília, Brasília, 2013.

MELO, R. A. A. Aspectos construtivos de túneis urbanos de baixa cobertura em solo: estudo de caso do túnel de acesso ao Centro Administrativo de Minas Gerais. Orientador: Paulo José Rocha de Albuquerque. 2013. 182f. Dissertação (Mestrado em Engenharia Civil) - Faculdade de Engenharia Civil, Arquitetura e Urbanismo - Universidade Estadual de Campinas, Campinas, 2013.

MILITITSK, J. Provas de cargas estática. In: SEMINÁRIO DE ENGENHARIA DE FUNDAÇÕES ESPECIAIS, 2., São Paulo. Anais [...]. São Paulo: ABMS, 1991, v. 2 p. 203-228.

MOURA, A. Análise de métodos semi-empíricos para o cálculo da capacidade de carga de estacas raiz de pequeno e grande diâmetro em obras de Fortaleza. Revista Tecnologia, Fortaleza, v. 32, n. 1, p. 56-72, 2011.

NIYAMA, S.; AOKI, N. Correlação entre as provas de carga dinâmica e estática no campo experimental da EPUSPIABEF. In: SEMINÁRIO DE ENGENHARIA DE FUNDAÇÕES ESPECIAIS, 2., São Paulo. Anais [...]. São Paulo: ABMS, 1991, v. 1, p. 285-293. 1991.

PERROTTA, M. M. et al. Mapa geológico do estado de São Paulo, escala 1:750.000. São Paulo: CPRM, 2005. (Programa Geologia do Brasil).

SHAO, G. Application of steel pipe pile composite soil nailing supporting combined foundation underpinning technology. In: INTERNATIONAL CONFERENCE ON GREEN MATERIALS AND ENVIRONMENTAL ENGINEERING, 2., [S. I.]. Proceedings [...]. Amsterdam: Atlantis Press, 2015.

SILVA, R. R. C. DA. Estacas injetadas autoperfurantes: metodologia de execução e determinação da sua capacidade de carga através de métodos semi-empíricos. Orientação: Marcos Massa Futai. 2011. 216p. Dissertação (Mestrado). Instituto de Pesquisas Tecnológicas, São Paulo, 2011.

SILVA, R. R. C. DA. Previsão da capacidade de carga em estacas raiz através de métodos semiempíricos associados a análises estatísticas. Revista CIATEC-UPF, Passo Fundo, v. 10, n. 2, p. 102$114,2018$.

SILVA, R. R. C. DA; PEREIRA, G. A. Verificação do aumento da capacidade de carga em estaca tipo raiz, através da aplicação de ar comprimido. Revista Ciência e Tecnologia, São Paulo, v. 20. p. 1-8, 2017. 
SCHLOSSER, F.; FRANK, R. Forever: fondations renforce'es verticalement: synthe'se des resultats et recommandations du Projet National sur les micropieux. Paris: Presse de l'ENPC, 2004.

VELLOSO, D. A.; LOPES, F. R. Fundações: fundações profundas. São Paulo: Oficina de Textos, 2010. v. 2.

\section{Sobre o autor}

\section{Rodrigo Rogério Cerqueira da Silva}

Pós-Doutorando pela Universidade Estadual de Campinas, Doutorado em inspeção de estruturas por ensaios não destrutivos pela Universidade Estadual de Campinas, mestrado pelo Instituto de Pesquisas Tecnológicas em fundações profundas, especialização em Docência do Ensino Superior pela Universidade Municipal de São Caetano do Sul, graduado pela Universidade Estadual de Campinas e Formação Pedagógica de Docentes pela Universidade Nove de Julho. Professor de engenharia civil da Universidade Nove de Julho (SP). Tem experiência na área de engenharia civil, com ênfase em fundações e obras de terra, tratamento de solos moles e inspeção de materiais e estruturas por meio de ensaios não destrutivos pelo método de ultrassom. Possui experiência na educação superior, atuando principalmente com aplicação de metodologias ativas de ensino-aprendizagem, aprendizagem baseada em problemas (PBL) e avaliação do desempenho de estudantes.

Recebido em: 17.04 .2020

Aceito em: 15.05.2020 